\title{
Réalisation d'un kart électrique performant : gestion de l'énergie embarquée et choix technologiques
}

\author{
Arnaud Sivert $^{1}$, F.Betin ${ }^{1}$, Thierry Lequeu ${ }^{2}$ : arnaud.sivert@iut.u-picardie.fr \\ (1) U.P.J.V, Laboratoire des Technologies innovantes (L.T.I), Institut Universitaire de Technologie de l'Aisne, Dept. GEII, 02880 SOISSONS \\ (2) Université François Rabelais de Tours, Institut Universitaire de Technologie de Tours, Département GEII, 37200 TOURS
}

\begin{abstract}
RESUME : Le véhicule électrique est un très bon système pédagogique car il peut être utilisé par tous les étudiants avec des couts moindres par rapport à la construction d'une voiture classique. Le challenge pédagogique de karting permet de motiver les étudiants et de mettre en œuvre différents choix technologiques. Ces choix sont souvent difficiles car il faut gérer correctement l'énergie embarquée, en accord avec les nouvelles technologies. En effet, ces véhicules du futur ont un compromis difficile entre le poids, la puissance, l'autonomie, et le prix. L'évolution des karts électriques a été rendue possible grâce aux moteurs qui fonctionnent sous faible tension et aux nouvelles batteries lithium. Cet article exposera la puissance résistante en fonction de la vitesse du véhicule, le couple accélérateur, les types de moteurs et variateurs utilisés, les types de sources d'énergies utilisés et les chargeurs. Enfin, l'exploitation pédagogique sera présenté car ce type de véhicule utilise de nombreux modules du génie électrique : convertisseur d'énergie, batterie, moteur, variateur, régulation, électronique, signalisation, instrumentation, capteur, mathématiques, mécanique...
\end{abstract}

Mots clés : kart électrique, challenge e-Kart, système pédagogique pluri technologique, électronique de puissance, instrumentation embarquée, batterie, super condensateur, moteur, chargeur, système de gestion de batterie.

\section{INTRODUCTION}

Pour réaliser un véhicule électrique, il faut faire un compromis entre la puissance, la consommation d'énergie, le poids, le volume, le prix et l'autonomie. Le kart électrique représente un bon support pédagogique pour mettre en œuvre ces compromis. Afin de donner un sens à cet objectif, un challenge national français de kart électrique est organisé par l'Association e-Kart. Il a lieu annuellement depuis 2006 et rassemble environ 40 karts de technologies différentes [7][8]. C'est l'occasion pour les étudiants de montrer leurs nouvelles idées et réalisations [3][6][9].

Nous allons voir dans ce papier, l'évolution de la puissance qui est demandée par un kart en fonction de la vitesse, le couple d'accélérateur pour démarrer rapidement. Les différents types de moteurs, de variateurs et de batteries seront passés en revue, ainsi que les chargeurs et l'instrumentation nécessaire à la mesure des grandeurs électriques, thermiques et mécaniques.

Les technologies utilisées dans ces karts sont identiques à celles présentes dans les petits véhicules électriques de La Poste ou du genre Twizy, dans les voiturettes de golf ou de parc d'attraction mais aussi en industrie dans les chariots de manutention.

Les karts électriques ont une faible nuisance sonore et sont de plus en plus utilisés pour la location au public sur des pistes intérieur («indoor»). Depuis 2009, la plupart de ces karts rivalisent en termes de performances avec les karts thermiques. Mais quels sont les caractéristiques des karts thermiques de compétition et quelles sont les motorisations électriques équivalentes?

\subsection{Les karts thermiques de compétition}

Les karts thermiques de compétition ont des moteurs de $125 \mathrm{~cm}^{3}$ qui développent au maximum des puissances d'environ $22 \mathrm{~kW}$ sans boite de vitesse, et jusqu'à $32 \mathrm{~kW}$ avec une boite de vitesse. Les karts sont par définition des engins qui n'ont pas de suspension, ni de différentiel. Le diamètre des roues est imposé à $25 \mathrm{~cm}$ (10 pouces). L'accélération est d'environ de $4.5 \mathrm{~s}$ pour atteindre $50 \mathrm{~m}$ et $6.5 \mathrm{~s}$ pour $100 \mathrm{~m}$. Les moteurs thermiques tournent à $18000 \mathrm{tr} / \mathrm{mn}$ ce qui entraine un entretien et une usure importante des pièces mécanique. La masse d'un kart thermique avec le pilote est d'environ $170 \mathrm{~kg}$. Nous allons à présent quantifier mathématiquement le kart électrique pour en connaître les caractéristiques (vitesse max, encombrement, accélération...). Dans un souci de simplicité, nous ne rentrerons pas dans le détail des pertes mécaniques du moteur, de la commande (régulation de vitesse ou de courant), de l'électronique de puissance...

Dans un premier temps, nous allons présenter sommairement la puissance résistante en fonction de la vitesse d'un kart.

\section{FORCE ET PUISSANCE RESITANTE EN REGIME ETABLI DE VITESSE}

Les forces résistantes du roulement dépendent du type de pneu, de la largeur du pneu et du type de chaussée. Elles sont représentées par le coefficient $\mathrm{k}_{\text {frottement }}$.

Les forces de roulement sont très faibles par rapport à la force de l'air qui dépend de la surface d'air S traversée, des turbulences donc du Cx.

Pour modéliser la puissance résistive d'un véhicule, l'équation suivante a été utilisée :

$\mathrm{P}_{\text {resistance }}(\mathrm{W})=\mathrm{k}_{\text {aero }} \cdot \mathrm{Vit}^{3}+\left(\mathrm{k}_{\text {frottement }}+\mathrm{k}_{\text {pente }}\right) \cdot \mathrm{Vit}$

Avec $\mathrm{k}_{\text {frottement }}$ en $\mathrm{W} /(\mathrm{km} / \mathrm{h})$ et $\mathrm{k}_{\text {aero }}$ en $\mathrm{W} /(\mathrm{km} / \mathrm{h})^{3}$

La force résistive de la pente, dépend de la masse du véhicule et du pourcentage de la pente. 
La puissance peut être observée en régime établi de vitesse sur la figure suivante [1][2]. Il faut $17 \mathrm{~kW}$ pour rouler à $120 \mathrm{~km} / \mathrm{h}$.

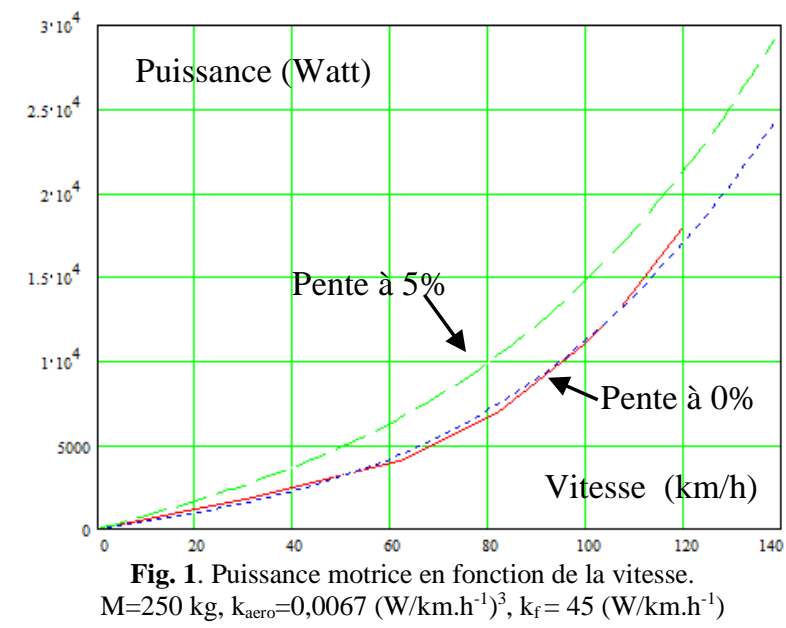

Maintenant, nous allons voir quel doit être le couple accélérateur que doit avoir le kart électrique.

\section{COUPLE D'ACCELERATION}

De par la présence des batteries, un kart électrique est plus lourd qu'un kart thermique. Sa masse avec pilote est de $250 \mathrm{~kg}$. S'il doit atteindre une vitesse de 100 $\mathrm{km} / \mathrm{h}$ en $4 \mathrm{~s}$, le couple accélérateur du moteur devra être supérieur à la valeur donnée par l'équation suivante :

$$
\mathrm{F}_{\text {moteur }}=\mathrm{M} \frac{\mathrm{dv}}{\mathrm{dt}}+\mathrm{F}_{\text {Resistive }}=1736 \mathrm{~N}+\mathrm{F}_{\text {Resistive }}
$$

Le couple que devra avoir le moteur dépendra de la valeur de la réduction de vitesse donc du choix de la vitesse maximale. Il y a donc un compromis entre la vitesse maximale (4) et le couple accélérateur (3) que peut fournir l'ensemble moteur/variateur.

$$
\begin{aligned}
& \mathrm{C}_{\text {moteur }}=\left(\mathrm{F}_{\text {moteur }} \cdot \text { ray on }_{\text {toue }}\right) \cdot \text { Re ducteur } \\
& \mathrm{V}_{\text {max }}(\mathrm{m} / \mathrm{s})=\mathrm{N}_{\text {maxmotor }} \cdot 2 \cdot \pi \cdot \text { Re ducteur } \cdot \text { Ray on }_{\text {roue }} / 60 \\
& \mathrm{~N}_{\text {maxmotor }}=\mathrm{U}_{\text {batterimax }} \cdot 60 /(\mathrm{k} \cdot 2 \cdot \pi) \\
& \mathrm{C}_{\text {motor }}=\mathrm{k} \cdot \text { Intensité }_{\text {motor }}
\end{aligned}
$$

Le choix de la transmission fait que l'on privilégie soit l'accélération, soit la vitesse maximale. Nous allons voir comment l'optimiser.

\subsection{Quel réducteur de vitesse choisir ?}

Les moteurs électriques ont en général une vitesse maximale d'environ $4000 \mathrm{tr} / \mathrm{mn}$. Donc, il faut un réducteur entre le moteur et l'arbre de transmission qui adapte la vitesse du karting et permet d'avoir un meilleur couple accélérateur. Des courroies crantées sont utilisées entre le moteur et l'arbre la transmission. Mais avec le niveau de couple accélérateur, la tension de la courroie crantée doit être importante pour qu'elle reste en prise avec les couronnes. Cette tension entraine des pertes non négligeables. Comme alternative à la transmission par courroie, il est possible d'utiliser des transmissions par chaine, plus bruyante.

Sur le tableau suivant, un exemple est fournit pour deux types de poulies de transmission et avec un kart disposant de 2 moteurs dont le courant est limité à 200A par les variateurs. Les moteurs ont une constante de couple ou de vitesse de $\mathrm{k}=0,17 \mathrm{Nm} / \mathrm{A}$ ou $\mathrm{V} / \mathrm{rad} / \mathrm{s}$ (5)(6). Ils fournissent un couple moteur maximal de $65 \mathrm{Nm}$.

Le tableau 1 donne la vitesse maximale, l'inertie ramenée sur l'arbre moteur, le temps pour atteindre $50 \mathrm{~m}$, ainsi que le courant en régime établi.

Tableau 1. Caractéristiques des moteurs en fonction du rapport de réduction,

\begin{tabular}{|ll|c|l|l|l|l|}
\hline $\mathrm{U}_{\text {batt }}$ & $\mathrm{I}_{\text {limit }}$ moteur & $\begin{array}{c}\text { Réduction } \\
\mathrm{D}_{\mathrm{m}} / \mathrm{D}_{\mathrm{a}}\end{array}$ & $\begin{array}{l}\text { Inertie } \\
\mathrm{kg} \cdot \mathrm{m}^{2}\end{array}$ & $\begin{array}{l}\mathrm{V}_{\max } \\
(\mathrm{km} / \mathrm{h})\end{array}$ & $\begin{array}{l}\mathrm{t}(50 \mathrm{~m}) \\
(\mathrm{en} \mathrm{s})\end{array}$ & $\begin{array}{l}\mathrm{I}_{\text {batterie (A) en ré- }} \\
\text { gime établi }\end{array}$ \\
\hline $72 \mathrm{~V}$ & $2 \times 200 \mathrm{~A}$ & $25 / 44$ & 1.26 & 112 & 5.3 & $210 \mathrm{~A}$ à $112 \mathrm{~km} / \mathrm{h}$ \\
\hline $72 \mathrm{~V}$ & $2 \times 200 \mathrm{~A}$ & $17 / 44$ & 0.58 & 75 & 4.4 & $70 \mathrm{~A}$ à $75 \mathrm{~km} / \mathrm{h}$ \\
\hline
\end{tabular}

La réduction de vitesse pour atteindre une certaine distance en un minimum de temps est déterminée à partir de l'équation (7) suivante :

$$
\text { reducteur }=\left[\text { Dis tance } \cdot \frac{\left(\text { Torque }_{\text {motor }}-\text { Torque }_{\text {load }}\right)}{\mathrm{N}_{\max }{ }^{2} \cdot\left(\frac{2 \cdot \pi}{60}\right)^{2} \cdot \text { ray on }_{\text {oue }}{ }^{3} \cdot \text { Masse }}\right]^{\frac{1}{3}}
$$

Pour simplifier le calcul, le couple de charge sera négligé par rapport au couple de démarrage.

Pour un kart évoluant sur une piste indoor avec des lignes droites de $50 \mathrm{~m}$ au maximum, il faut une réduction de 13/44. Si l'on veut réellement optimiser la transmission en fonction d'une piste, il faudrait donc une boite de vitesse.

Avec le tableau précédent et pour un réducteur de 17/44, on peut voir l'accélération et le régime établi du moteur sur la figure 2. Le couple de freinage est inférieur ou égale à ce que peut faire le moteur. Donc, ce type de freinage électrique est relativement long et il est peut utilisé en course. En effet, sur circuit, il est préférable de freiner tardivement et énergiquement.

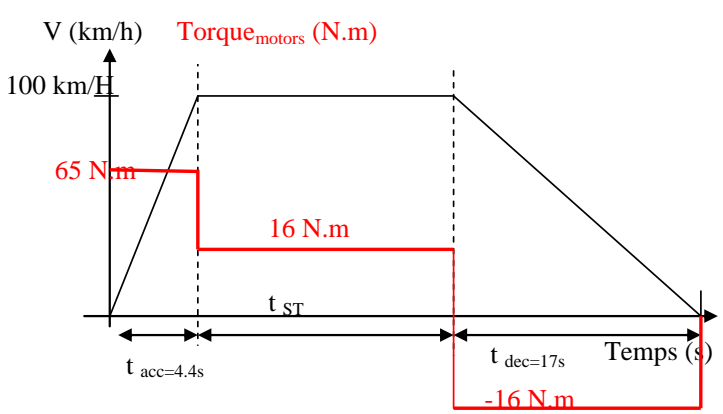

Fig 2. Vitesse et couple demandés aux moteurs avec reducteur 17/44 sur banc de charge. 
Voyons maintenant l'impact de ce régime de fonctionnement sur la température du moteur.

\subsection{Et la température du moteur ?}

En fonction du paramétrage de la limitation du courant et du nombre d'accélération, la température du moteur va s'élever.

Il faut donc déterminer le courant équivalent thermique du moteur pendant un cycle de fonctionnement (un tour de piste) à partir de l'équation suivante :

$I_{\text {m equ }}=\sqrt{\frac{\sum_{i=1}^{n}\left(I_{n}\right)^{2} \cdot t_{i}}{\sum_{i=1}^{n} \cdot t_{i}}}$

Le courant équivalent thermique doit être plus faible que le courant nominal du moteur.

\begin{tabular}{|c|c|c|c|c|c|c|c|c|c|}
\hline \multicolumn{10}{|c|}{ Tableau 2. Différents types de moteurs } \\
\hline Type de moteur & $\begin{array}{c}\text { Tension } \\
\text { max }\end{array}$ & $\begin{array}{c}\mathrm{I}_{\text {nom }} \\
\mathrm{A}\end{array}$ & $\mathbf{I}_{\max }$ & $\begin{array}{l}\mathrm{P}_{\mathrm{mot}} \\
\mathrm{kW}\end{array}$ & $\begin{array}{c}\text { Poids } \\
\text { kg }\end{array}$ & $\begin{array}{l}\text { Long* } \varnothing \& \\
\text { volume } \mathrm{cm}^{3}\end{array}$ & $\begin{array}{c}\text { Prix en } \\
2012\end{array}$ & $\begin{array}{c}\mathrm{N}_{\max } \\
\mathrm{Tr} / \min \end{array}$ & IP \\
\hline MCC (avec balais) & $72 \mathrm{~V}$ & 190 & 400 & 14 & 11 & $11 * 20=>690$ & $1600 €$ & 4000 & 22 \\
\hline Synchrone & $48 \mathrm{~V}$ & 300 & 400 & 14 & 2 & $8.5^{*} 11=>290$ & $1300 €$ & 8000 & 22 \\
\hline Asynchrone & $28 \mathrm{~V}$ & 150 & 300 & 10 & 20 & $30 * 18=>1700$ & $1500 €$ & 4500 & 55 \\
\hline
\end{tabular}

Les moteurs DC sont associés à un hacheur qui limite le courant de démarrage. Ces moteurs et variateurs ont un encombrement réduit ce qui permet de mettre 2 moteurs sur le même arbre de transmission afin d'additionner le couple des 2 moteurs. Souvent 2 hacheurs sont utilisés qui permettent aux moteurs de fournir le même couple en fonction de la consigne d'accélération.

On peut voir sur la courbe expérimentale suivante la tension et le courant d'un kart électrique bi-moteur en fonction du temps, avec un réglage du courant à $250 \mathrm{~A}$ par moteur.

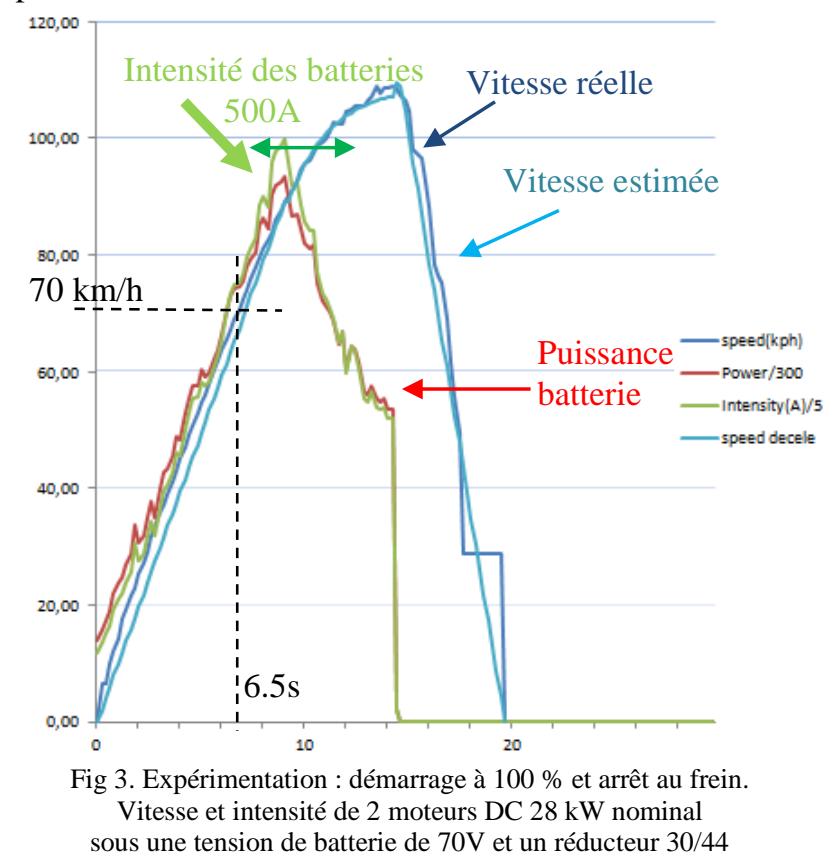

Sur la figure 3, le couple des moteurs est de $80 \mathrm{Nm}$ pour 500A car la constante de couple de nos moteurs est de $0,17 \mathrm{Nm} / \mathrm{A}$. Le couple de pertes mécaniques correspond à $2,5 \mathrm{Nm}$ avec un courant à vide de $15 \mathrm{~A}$. On vérifie que
A cause des accélérations successives que la piste occasionne, le courant nominal peut être vite dépassé et détruire le moteur. Les motorisations utilisées sur les karts électriques de compétition demandent d'une ventilation forcée qui consomme quelques centaines de watts.

Maintenant que la puissance demandée ainsi que le choix du réducteur est connu, nous allons voir les différents types de moto-variateurs.

\section{TYPES DE MOTEUR ET DE CONTRO- LEUR}

Trois types de moteurs sont utilisés sur les karts et présentés sur le tableau 2 : le couple de perte est négligeable devant le couple de démarrage des 2 moteurs.

Même si l'équation différentielle (2) n'est pas linéaire, l'évolution de la vitesse en fonction de temps peut être modélisée simplement par l'équation (9) suivante :

vitesse $(t)=$ vitesse $_{\text {finale }}+\left(\right.$ Vitesse $_{\text {initiale }}-$ Vitesse $\left._{\text {finale }}\right) \cdot e^{\frac{-t}{\tau}}$

Avec $\tau$ la constante de temps. Si la vitesse initiale est nulle, la constante de temps correspond à $63 \%$ de la vitesse finale.

A $63 \%$ de la vitesse finale (ici $110 \mathrm{~km} / \mathrm{h}$ ), la vitesse du kart est de $70 \mathrm{~km} / \mathrm{h}$. Le temps de réponse est de $6,5 \mathrm{~s}$.

Les moteurs DC demandent un entretien et sont de plus en plus remplacés par des moteurs sans balais («brushless ») qui permettent d'avoir encore moins d'encombrement que le moteur DC, et ne nécessitent pas d'entretien des balais. Pas contre, le variateur est plus complexe qu'un hacheur. En effet, il faut un onduleur triphasé pour alimenter les bobines en fonction du capteur de position du rotor.

Le moteur asynchrone présenté au tableau 2 et son variateur sont relativement encombrant et il est peu commode d'installer 2 moteurs. Le variateur du moteur asynchrone est une commande vectorielle : il permet d'avoir un maximum de couple au démarrage et retenir le véhicule à vitesse nulle sans utiliser de frein mécanique à manque de courant. Le moteur asynchrone avec sont indice de protection IP 55 est idéal pour les karts de location ou les applications industrielles.

Tous les variateurs peuvent retourner l'énergie aux batteries. Mais il faut faire attention que les batteries puissent recevoir cette énergie sans être détruites. Nous allons maintenant voir les différents types de batteries couramment utilisés sur les karts électriques. 
BATTERIES

La solution standard d'alimentation électrique d'un kart est d'utiliser 4 batteries $12 \mathrm{~V}$ au plomb afin d'avoir $48 \mathrm{~V}$. Avec une puissance de $10 \mathrm{~kW}$, l'autonomie est d'environ 10 minutes, avec un temps de recharge de 10 minutes. Les prototypes de kart à 2 moteurs qui demandent $54 \mathrm{~kW}$ au démarrage et seulement de 10 à $30 \mathrm{~kW}$ en nominal sont alimentés sous $72 \mathrm{~V}$, dans le but de minimiser l'intensité demandée aux batteries. En effet à partir du tableau 3, on peut observer que plus le taux de décharge est important et plus le prix de l'accumulateur sera élevé. De même, nous pouvons observer l'encombrement, le poids et la capacité énergétique en fonction du type de source d'énergies.

La figure 4 présente 2 karts ayant la même motorisation bi moteur mais avec 2 types de batteries différentes. Avec l'accumulateur Li-fer, le poids est divisé par 2 pour une autonomie multiplié par 4. Ce kart électrique Li-fer a une autonomie d'environ 30 minutes à 110 $\mathrm{km} / \mathrm{h}$.

Pour l'instant, aucun prototype n'est passé en 400V avec 100 éléments de 20 Ah en série pour un coût batterie de 5000€. Ceci permettrait d'utiliser des motovariateurs industriels [5]. Les principaux inconvénients sont la sécurité électrique, l'encombrement et la gestion de tous les éléments au travers d'un BMS («Batterie Management System » Système de Gestion des batteries).

\begin{tabular}{|l|l|r|l|l|l|l|}
\hline \multicolumn{7}{|c|}{ Tableau 3. Caractéristiques des batteries } \\
\hline $\begin{array}{l}\text { Type de batteries } \\
\text { pour 72V }\end{array}$ & $\begin{array}{l}\text { Energie } \\
\text { Wh }\end{array}$ & $\begin{array}{l}\text { Taille en cm \& } \\
\text { Volume total en } \mathrm{dm}^{3}\end{array}$ & $\begin{array}{l}\text { Mass } \\
\text { e } \\
\mathrm{kg}\end{array}$ & $\begin{array}{l}\text { Prix en } \\
2013\end{array}$ & $\begin{array}{l}\text { Taux } \\
\text { charge } \\
\max \end{array}$ & $\begin{array}{l}\text { Taux } \\
\text { décharge } \\
\text { max }\end{array}$ \\
\hline Plomb 6S 20Ah 6S & 1440 & $6 \times 20 * 17,5 * 24=>50 \mathrm{dm}^{3}$ & 100 & $1600 €$ & $100 \mathrm{~A}=5 \mathrm{C}$ & $400 \mathrm{~A}$ \\
\hline Li-Fe 90Ah 22S & 6480 & $22 \times 14 * 6 * 22=>41 \mathrm{dm}^{3}$ & 68 & $2800 €$ & $90 \mathrm{~A}=1 \mathrm{C}$ & $300 \mathrm{~A}=3 \mathrm{C}$ \\
\hline Li-Po 100Ah 18S & 7200 & $35 \mathrm{dm}^{3}$ & 55 & $7000 €$ & $100 \mathrm{~A}=1 \mathrm{C}$ & $500 \mathrm{~A}=5 \mathrm{C}$ \\
\hline Super cap. 3000F 36S & 66 & $36 \times \varnothing 6.4 * 13.8 * 36=>23 \mathrm{dm}^{3}$ & 18 & $1800 €$ & $147 \mathrm{~A}$ & $2165 \mathrm{~A}$ \\
\hline
\end{tabular}

Par exemple, si vous voulez aller plus vite alors la puissance motrice sera plus importante, donc le moteur sera plus lourd et plus cher, tout comme les batteries. Il faudra utiliser davantage de capacité énergétique, voire des technologies plus performantes et votre budget en subira les conséquences. L'encombrement de l'énergie embarquée est limité sur ce type de véhicule comme on peut le voir sur la figure suivante.

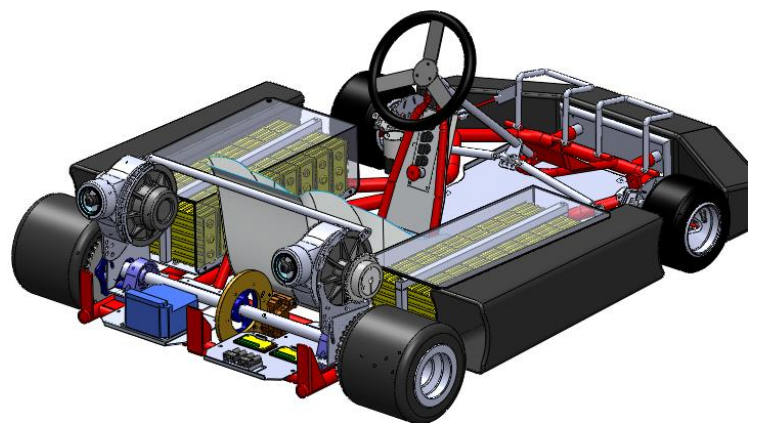

Fig. 5 - Etude du centre de gravité, du CX, du refroidissement des moteurs et des protections sur SolidWorks avant réalisation.

Une autre difficulté est de disposer de chargeurs rapides et sécurisés pour les batteries.
Le tableau 3 indique qu'un taux de décharge important provoque une augmentation du prix de la batterie et réduit la durée de vie de la batterie. Le prix et la masse du kart va dépendre énormément de la technologie de l'accumulateur.

Par conséquent, trouver le compromis idéal pour un kart électrique n'est pas une chose aisée, car tous les éléments du cahier des charges sont liés.

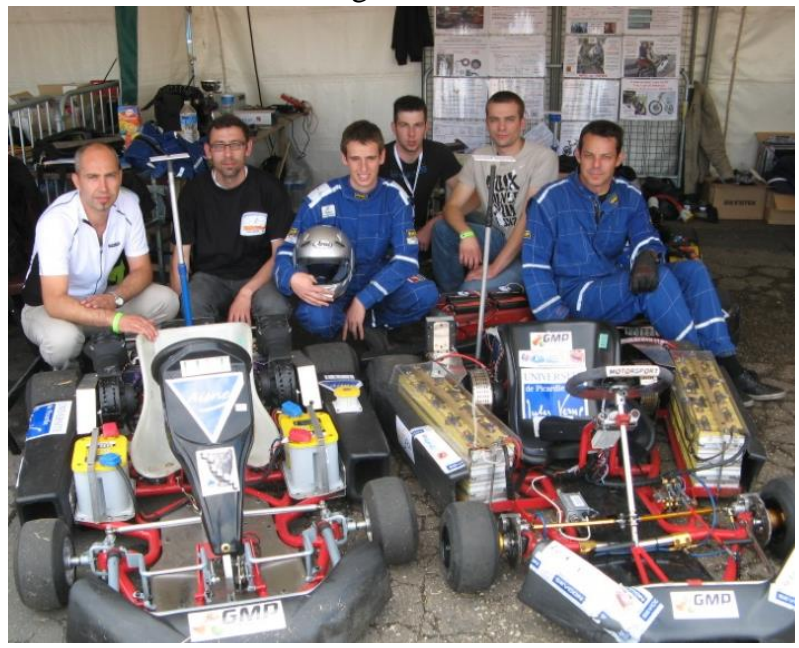

Fig 4. Karts électriques 2 moteurs $28 \mathrm{~kW}$ nominal : $48 \mathrm{~V}$ au plomb à gauche et Li-fer à droite $72 \mathrm{~V}$. Challenge National Vierzon e-Kart 2011.

\section{CHARGEURS ET BMS}

Une des épreuves du challenge pédagogique est la course d'endurance de 2 heures avec 2 karts. Pendant que l'un des karts roule, l'autre est en charge. L'autonomie des batteries et surtout leur capacité de recharge sont très importantes pour cette épreuve. En effet, il faut charger autant que l'on consomme et il faut des chargeurs qui puissent fournir des courants importants. Plus les chargeurs peuvent fournir un courant important et plus ils ont chers et difficile à réaliser. Les accumulateurs se chargent à courant constant, puis à tension constante, comme on peut le voir sur la figure suivante :

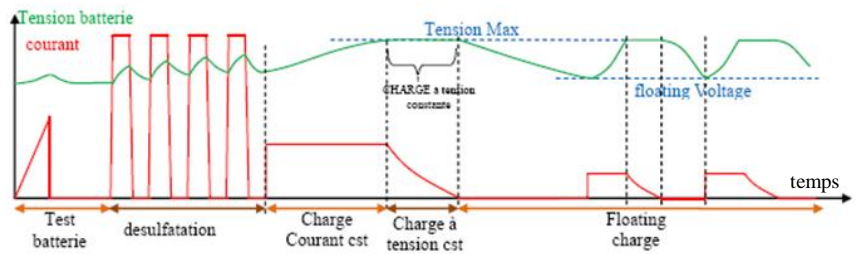

Fig. 5 - Étapes de fonctionnement du chargeur [4].

Pour les accumulateurs Lithium, il faut en plus vérifier que la tension de seuil de chaque élément ne soit pas jamais dépassée et surveiller la température. La gestion 
de ces tensions est réalisée par le circuit BMS (Battery Management System). Le courant de charge doit diminuer jusqu'à ce que le courant soit nul pour qu'aucune tension de seuil d'un des éléments ne soit pas dépassée. Donc, s'il y a un élément qui est chargé à $100 \%$ et que le courant devient nul, alors les autres éléments seront chargés à une valeur inferieure à $100 \%$ donc, il y aura un déséquilibre de charge.

Donc toutes les 10 charges, il est préférable de charger les éléments de façon indépendante pour que tous les éléments soient chargés à $100 \%$ (équilibrage). Étant donné qu'il y a un grand nombre d'éléments, il faut beaucoup de chargeurs indépendants mais il est possible de charger lentement avec un courant de 1/10 de la capacité énergétique. Cette solution reste encore relativement chère, c'est pourquoi de nombreux constructeurs proposent des équilibreurs avec résistance qui dévient le courant de charge des éléments les plus chargés. Mais le courant dévié est faible, seulement de 0,3 A à $0,5 \mathrm{~A}$.

La figure 6 montre la disparité entre les courants de charge ainsi que la tension de chaque élément en fonction du temps : un élément est chargé à la tension de seuil de 3,7V (100\%), et les autres éléments sont déséquilibrés. Sur les éléments Li-Fe, après une charge à $100 \%$, toutes les tensions des éléments redescendent à $3.3 \mathrm{~V}$, donc il y a le phénomène de «floating charge » (charge d'entretien), comme sur une batterie au plomb.

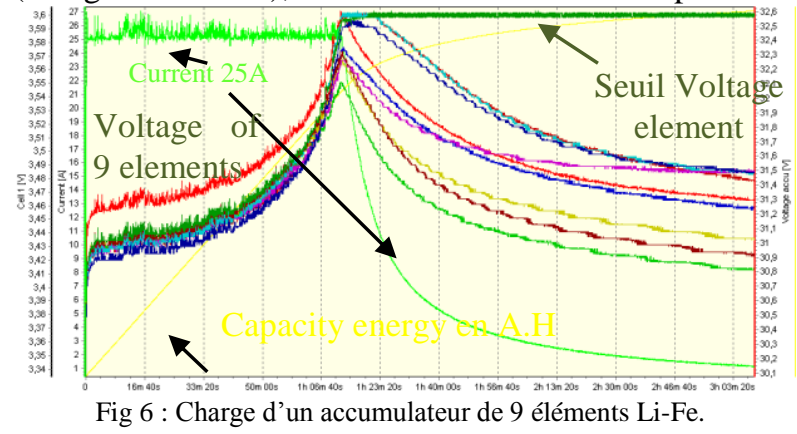

Les BMS doivent protéger chaque élément de l'accumulateur contre :

- les sur-tensions pendant la charge,

- les sur-tensions pendant les freinages,

- les sous-tensions pendant la décharge (inutile, vous ne pouvez plus rouler, mais utile si vous oubliez d'éteindre le variateur). En effet, si vous déchargez à $100 \%$, un accumulateur lithium, il va se mettre en court circuit et sera détruit. Le BMS doit indiquer lorsque la capacité énergétique est inférieure à $15 \%$.

- les échauffements, mais il est difficile à mettre en œuvre car il faut mesurer la température de chaque éléments.

La réalisation d'un BMS n'est pas une chose facile et coûte entre la moitié et $100 \%$ du prix d'un accumulateur. Nous allons voir la nécessité (ou non) des fonctions du BMS :

- Si le variateur ne freine pas électriquement, il n’y a pas besoin d'embarquer la détection de la tension haute des cellules, car il est possible d'intégrer au chargeur la surveillance de toutes les tensions des éléments. Par conséquent, la tension de seuil de chaque élément ne peut être dépassée et le chargeur effectue le rééquilibrage.

- En revanche, avec un chargeur de batterie sans équilibrage, il faudra un BMS, qui permet de dévier le courant de l'élément qui sera chargé à $100 \%$. Mais en général, ces BMS ne permettent pas des charges élevées et des freinages électriques importants.

- Si vous avez chargé à $100 \%$ la batterie à une température de $18^{\circ} \mathrm{C}$, et si vous la laissez en plein soleil à $50^{\circ} \mathrm{C}$, la tension de vos cellules va dépasser la tension de seuil et votre accumulateur risque de s'enflammer. Le BMS devra décharger vos batteries avant que cela ne se produise.

- Si vous avez un élément qui est en fin de vie et qui devient instable thermiquement, le BMS peut couper l'alimentation grâce à de nombreux capteurs de température et empêchera que votre pack de batterie ne prenne feu.

- Les prototypes de kartings utilisent souvent différentes tensions prélevées sur les cellules intermédiaires de la batterie pour la signalisation et l'instrumentation. Ce principe déséquilibre les éléments et oblige un ré-équilibrage avec le BMS mais il faut que celui-ci puisse supporter un courant de charge important (ou de la patience...).

- Les BMS peuvent communiquer avec les variateurs et demander à limiter le taux de décharges de l'accumulateur, si un des éléments est en soustensions.

A partir du tableau 3, on peut voir que le taux de décharge des batteries est faible par rapport au courant qui peut être supporté par les moto-variateurs. L'utilisation de super condensateurs peut être envisagée pour fournir le courant impulsionnel nécessaire à chaque accélération, ainsi que la récupération du courant de freinage.

\section{LES SUPERCONDENSATEURS}

Les super-condensateurs présentés au tableau 3, permettent de fournir de fortes intensités mais ils ont une faible capacité énergétique par rapport à une batterie. Un autre avantage de ce composant est d'avoir un nombre de cycle de charge et de décharge 1000 fois supérieure à celui d'une batterie. Ils restent assez chers malgré une baisse des tarifs ces dernières années.

Il est donc intéressant d'associer les avantages électriques des super-condensateurs et de la batterie.

Par contre, un compromis entre le volume, le poids et un prix acceptable doit être trouvé. Il faut aussi gérer la charge et la décharge des deux sources d'énergie par l'utilisation de convertisseurs spécifiques qui doivent limiter le courant sortant et entrant des batteries et des super-condensateurs. Ces convertisseurs doivent aussi surveiller et limiter la tension maximale de chaque 
élément de la batterie et des super-condensateurs (fonction B.M.S.).

Une solution est que la batterie et les supercondensateurs fournissent l'énergie à un bus continu DC par l'intermédiaire de deux hacheurs réversibles en courant (hacheur 1 et hacheur 2) appelé «alimentation hybride »[10]. Ce bus continu permet d'alimenter l'ensemble variateur-moteur du véhicule.

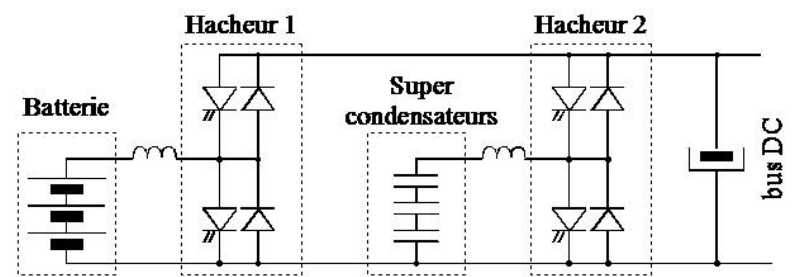

Fig. 7. Présentation de l'« alimentation hybride » batterie et super condensateurs [10]

Une autre solution consiste à mettre en les supercondensateurs en parallèle de chaque élément de la batterie Lithium. Comme les résistances internes des super-capacités sont bien inférieures à celle de la batterie, le courant de pointe sera fourni en majorité par les super condensateurs. Cet assemblage réduit les cycles de charge et de décharge des cellules Lithium et augmente leur durée de vie. Pour supporter la tension maximale de 3.7 à $4 \mathrm{~V}$ d'une cellule Lithium, il faut associer 2 super condensateurs de $2.7 \mathrm{~V}$ en série. Ainsi, la tension de seuil des super-condensateurs ne sera jamais dépassée (si le BMS protège correctement la cellule Lithium). Pour que la mise en parallèle se passe bien, il faudra veiller à charger les super-condensateurs à la même tension que les éléments de l'accumulateur afin de limiter les courants de circulation qui peuvent être très important.

Après avoir présenté les solutions techniques, nous allons voir comment le kart électrique peut être exploité pédagogiquement.

\section{EXPLOITATION PEDAGOGIQUE}

Le challenge pédagogique de kart permet d'avoir une motivation des étudiants pour étudier, réaliser, améliorer et monter sur un ou plusieurs podiums.

Dans un véhicule, tous les modules du génie électrique sont utilisés, comme par exemple :

- la gestion de l'énergie embarquée avec les batteries et les super condensateurs ;

- l'électronique de puissance via les chargeurs, variateurs, BMS, coupe circuit, signalisation à LED...

- l'électronique avec l'instrumentation embarquée pour la mesure de vitesse, de distance, de capacité énergétique, de température et l'envoie des données dans les stands ;

- l'informatique pour la gestion des données;

- la micro-informatique avec la programmation des microcontrôleurs dans l'instrumentation, les variateurs, les BMS, les chargeurs...

- l'automatisme pour régulation des courants moteur et chargeur et la régulation de la tension des chargeurs ;
- l'automatique avec l'utilisation d'automate haute vitesse pour la commande du variateur ;

- la mécanique lors de l'étude de la dynamique des systèmes, freins, réalisation de carénage en matière composite pour minimiser le CX...

- la thermique avec l'étude des échauffements des composants du kart électrique.

La liste des possibilités technologiques et pédagogiques est grande et n'est pas exhaustive. Elle permet de faire des enseignements pluri technologiques.

\section{CONCLUSION}

L'étude et la réalisation d'un véhicule électrique est un très bon support pédagogique pour nos étudiants de génie électrique et mécanique.

Cet article a permis de prouver que les karts électriques ont les mêmes dynamiques que les karts thermiques de compétition, avec une autonomie honorable (30 minutes). De même, le temps de charge est très court, de l'ordre de 10 minutes pour des batteries au plomb et inférieur à une heure pour les batteries Lithium.

Le prix d'un kart électrique est compris entre 4000 et 9000 euros en fonction de technologies utilisées. Ce qui correspond aux prix d'un kart thermique de compétition.

Le prix de la consommation électrique est 5 fois plus faible que celui de l'essence et le moteur électrique demande bien moins d'entretiens que son homologue thermique.

Les étudiants sont très motivés avec ce type de projet car ils se sentent utiles en répondant à une demande de notre société qui veut minimiser notre impact sur la planète et améliorer notre futur énergétique.

\section{Bibliographie}

[1] A. SIVERT, T. LEQUEU, "Je construis mon véhicule électrique", édition Dunod, 2013, 140 pages.

[2] A. SIVERT, F. BETIN, J.P. BECAR, T. LEQUEU, "Do electric go-karts are getting better than gas powered ones?", Conference EVER MONACO, mars 2012. 6 pages.

[3] J.P. BECAR, T. LEQUEU, S. COLTON, "An Electrical Go-Kart for Project Based Learning Platform" EVER 2011.

[4] A. SIVERT, F. BETIN, J.P. BECAR, "A Fuzzy Logic Application for Go-Kart: a Battery Charger", EVER 2011.

[5] E. ARMANDO, B. BOAZZO, P. GUGLIELMI, "BullET: an High Performance Electric Kart”, EVER 2011.

[6] T. LEQUEU, B. BIDOGGIA, Y. DERRIEN, N. GODEFROY, "Two Examples of Pedagogical Applications of Electrical Go-Karts", EPE 2007, Denmark, 9 pages.

[7] Site web de l'Association e-Kart : http://www.e-kart.fr.

[8] Site web des réalisations de véhicules électriques de l'IUT GEII de l'Aisne : http://aisne02geii.e-kart.fr/, 2014.

[9] IUT en ligne http://www.iutenligne.net/ressources/unsupport-pedagogique-pluritechnologique-le-kartelectrique.html, 2013.

[10] E. HOANG, B. REVOL, G. FELD, "Stockage d'énergie portable hybride - Association accumulateur électrochimique - super condensateur - Mise en application sur un vélo électrique”, CETSIS-EEA 2005, 7 pages. 